\title{
EFICIÊNCIA DA SUBSOLAGEM NA PROFUNDIDADE DE PREPARO DO SOLO EM FUNÇÃO DA DECLIVIDADE DO TERRENO
}

\author{
Daniel Pena Pereira ${ }^{1}$, Nilton César Fiedler ${ }^{2}$, Julião Soares de Souza Lima ${ }^{3}$, \\ Pompeu Paes Guimarães ${ }^{4}$, Rômulo Môra ${ }^{4}$, Flávio Cipriano de Assis do Carmo ${ }^{5}$
}

(recebido: 21 de setembro de 2010; aceito: 29 de junho de 2012)

\begin{abstract}
RESUMO: Nesta pesquisa objetivou-se avaliar a eficiência da subsolagem por meio da medição das profundidades atingidas em diferentes classes de declividades do local em estudo. Utilizou-se um subsolador-adubador de arraste com haste única lisa parabólica e ponteira não alada, com dimensões de $1 \frac{1 / 2}{2}$ polegadas de espessura e $320 \mathrm{~mm}$ de largura tracionado por um trator com motor de potência bruta de aproximadamente $112 \mathrm{~kW}$, com tração dianteira auxiliar ativada e bitola regulada para a máxima largura entre rodas Executou-se essa operação em função de sete classes de declividade. A inclinação lateral máxima para efetuar a subsolagem foi calculada de acordo com o centro de gravidade e a bitola do trator. Determinou-se o número e a amplitude das classes pela fórmula de Sturges. Os dados foram avaliados segundo a análise de regressão para o caso de dados com repetição a $5 \%$ de significância. O modelo de regressão proposto foi adequado para descrever os valores uma vez que teve resultado significativo para o teste F. Para o ajuste da equação de regressão, o coeficiente de determinação apresentou como resultado 78,95\%, indicando que esse valor representa a quantidade de valores de profundidade que são explicados pela declividade. Assim, pode-se afirmar que existe tendência para a profundidade da subsolagem diminuir com o aumento da declividade do terreno, sendo um limitador para a qualidade da operação de preparo mecanizado do solo. Demonstrou que as declividades até $40 \%$ permitiram atingir a profundidade mínima de $0,50 \mathrm{~m}$ para a subsolagem.
\end{abstract}

Palavras-chave: Manejo florestal, compactação do solo, profundidade de trabalho.

\section{EFFICIENCY OF SUBSOILING DEPTH ACCORDING TO THE SLOPE OF THE LAND}

\begin{abstract}
The effectiveness of subsoiling by measuring the depths achieved in different classes of slope of a forest plantation was evaluated. This operation was made with a fertilizer trawling subsoiler with a single smooth parabolic rod depending on seven groups of slope and the maximum lateral inclination of the tractor to perform the subsoiling. It was determined the number and breadth of slope classes by Sturges formula. Data were assessed by regression analysis for data with repetition at $5 \%$ significance level. The proposed regression model was adequate to describe the values given that it presented significant result for the F test. For the adjustment of the regression equation, the coefficient of determination was $78.95 \%$, representing the the depth values that are explained by the slope. Thus, it can be said that the depth of subsoiling decreases as the steepness of the ground increases and is a limiter for the quality of the mechanized soil preparation. The results demonstrate that slopes up to $40 \%$ allowed the operation of subsoiling to reach the minimum depth of $0.50 \mathrm{~m}$ for forest cultivation.
\end{abstract}

Key words: Forest management, soil compaction, depth of subsoiling.

\section{INTRODUÇÃO}

Até o final da década de 80, o preparo de solo nos locais com reflorestamento consistia na eliminação, em geral por queima, dos resíduos da vegetação anterior e no revolvimento intenso de todo o solo da camada superficial, à semelhança do que se utilizava nos cultivos agrícolas convencionais (MOLIN; SILVA JÚNIOR, 2003). O crescimento inicial da muda era rápido, o que levava à falsa impressão de excelente e duradoura condição para as

\footnotetext{
${ }^{1}$ Engenheiro Agrônomo, Doutorando em Produção Vegetal - Universidade Federal do Espírito Santo/UFES - Programa de Pósgraduação em Produção Vegetal - Alto Universitário, s/nº - Guararema - Cx. P. 16 - 29500-000 - Alegre, ES, Brasil - daniel@geraes.org ${ }^{2}$ Engenheiro Florestal, Professor Doutor em Ciência Florestal - Universidade Federal do Espírito Santo/UFES - Centro Agropecuário Departamento de Engenharia Florestal - Alto Universitário, s/nº - Guararema - Cx. P. 16 - 29500-000 - Alegre, ES, Brasil fiedler@pq.cnpq.br

${ }^{3}$ Engenheiro Agrícola, Professor Doutor em Ciência Florestal - Universidade Federal do Espírito Santo/UFES - Centro Agropecuário Departamento de Engenharia Florestal - Alto Universitário, s/nº - Guararema - Cx. P. 16 - 29500-000 - Alegre, ES, Brasil limajss@yahoo.com.br

${ }^{4}$ Engenheiro Florestal, Doutorando em Engenharia Florestal - Universidade Federal do Paraná/UFPR - Av. Pref. Lothário Meissner, 900 Jardim Botânico - Campus III - 80210-170 - Curitiba, PR, Brasil - pompeupaes@yahoo.com.br, romulo_floresta@hotmail.com ${ }^{5}$ Engenheiro Florestal, Mestrando em Ciências Florestais - Universidade Federal do Espírito Santo/UFES - Alto Universitário, s/n ${ }^{\circ}$ Guararema - Cx. P. 16 - 29500-000 - Alegre, ES, Brasil - flaviocipriano@ hotmail.com
} 
plantas. $\mathrm{Na}$ tentativa de solucionar os problemas decorrentes do preparo convencional do solo, para implantação de povoamentos florestais surgiram os preparos conservacionistas, que proporcionam menor mobilização do solo e mantêm maior proteção da superfície com os resíduos culturais. O plantio direto e o cultivo mínimo são tipos de manejo conservacionistas que procuram minimizar essa mobilização (RICHART et al., 2005).

A tecnologia atualmente utilizada no preparo do solo, para plantios florestais tecnificados, utiliza o cultivo mínimo, realizando um preparo de solo localizado, apenas na linha da cova de plantio, sendo que a subsolagem e o coveamento são as principais operações de preparo do solo desse sistema (GATTO et al., 2003; GONÇALVES et al., 2000).

A compactação do solo é um processo não-natural em que a porosidade e a permeabilidade são reduzidas, a sua resistência é aumentada e muitas mudanças são provocadas em sua estrutura e em várias características de seu comportamento (RICHART et al., 2005). Para Fessel (2003), a subsolagem é utilizada para romper camadas de solo impermeáveis ou compactadas, em profundidades maiores do que $0,40 \mathrm{~m}$, promovendo a infiltração de água, pouca mistura e nenhuma inversão. Atualmente, profundidades até $0,35 \mathrm{~m}$ são consideradas como operação de escarificação. Especificamente no setor florestal, Dalbem et al. (2008), recomendam a profundidade mínima de 0,50 m no preparo mecanizado de solo, para plantio de eucalipto.

Apesar de existirem diversas opções para realizar o preparo do solo, a subsolagem se consolidou no setor florestal, pelos seus efeitos benéficos para o desenvolvimento das plantas (BLAZIER; DUNN, 2008; DEDECEK et al., 2007; MINATEL et al., 2006; RAPER et al., 2009) e as suas vantagens operacionais, tais como maior capacidade de trabalho e menor custo (RAPER; BERGTOLD, 2007; SALVADOR et al., 2008).

Os resultados obtidos por Gatto et al. (2003) mostram que as florestas de eucalipto cultivadas com preparo de solo mecanizado, por meio da subsolagem, podem atingir maiores produtividades, com reflexos no volume de madeira. Outros estudos devem ser dirigidos a fim de definir limites seguros para trafegabilidade do conjunto trator-subsolador e obter eficiência ao atingir profundidades mínimas exigidas para a operação de subsolagem, em função da declividade do terreno e segurança para o operador.

Cerne, Lavras, v. 18, n. 4, p. 607-612, out./dez. 2012
A realização de atividades agrícolas mecanizadas em locais de topografia acidentada exige atenção às normas de segurança que, dentre outros, considera-se a inclinação do terreno e a estabilidade lateral das máquinas utilizadas (CHISHOLM, 1979; MASHADI; NASROLAHI, 2009). A declividade é um fator limitante para o uso seguro de tratores agrícolas em terreno íngreme, e tanto o conhecimento do ângulo máximo de estabilidade lateral de tratores quanto do zoneamento agrícola onde as operações são realizadas, são fatores importantes para o gerenciamento do preparo mecanizado de solo. A perda de estabilidade é um fator que contribui frequentemente para a capotagem de tratores por essas terras (GIALAMAS et al., 2006; GILFILLAN, 1967; HUNTER, 1981; SPENCER, 1978) e o conhecimento dos limites de cada máquina, evitam-se acidentes, no caso de tombamentos do trator, com perigo aos operadores e podendo causar danos às máquinas e implementos.

Em função do exposto, objetiva-se, por meio dessa pesquisa, estudar a eficiência do preparo mecanizado de solo em plantio florestal, em relação à profundidade de trabalho em diferentes classes de declividade.

\section{MATERIAL E MÉTODOS}

\section{1 Área de estudo}

Esta pesquisa foi executada na propriedade rural Fazenda Cachoeira Bonita localizada no município de São José do Calçado, entre as coordenadas UTM (Projeção Universal Transversa de Mercator - DATUM SAD-69 Fuso 24S): norte $=7.682 .974,189 \mathrm{~m}$; sul $=7.682 .588,817 \mathrm{~m}$; leste $=226.675,383 \mathrm{~m}$; e oeste $=226.119,654 \mathrm{~m}$, extremo sul do estado do Espírito Santo.

Pelo método de Köppen, essa região é caracterizada pelo tipo climático $C w a$ - descrito como: $\mathrm{C}=$ mesotérmico, $\mathrm{w}=$ chuvas nos verões e seca no inverno; $\mathrm{e} \mathrm{a}=$ temperatura média do mês mais quente é superior a $22^{\circ} \mathrm{C}$. O local de estudo apresenta precipitação média anual de $1380 \mathrm{~mm}$ por ano e a temperatura média de $20,1^{\circ} \mathrm{C}$ (PEREIRA, 2010).

$\mathrm{O}$ estudo foi desenvolvido em local com preparo mecanizado do solo e com uma inclinação média do terreno de 36,5\%. Segundo o mapa geral de solos do Espírito Santo, baseado no mapa exploratório de solos do Projeto RADAMBRASIL (LANI, 2008), o solo predominante é Latossolo Vermelho-Amarelo (LVA), do tipo LVa9. É olocal da grande área de solos amarelos, profundos, pobres e apresenta teores granulométricos médios de $51 \%$ de argila, $5 \%$ de silte e $44 \%$ de areia, no perfil de $0-20 \mathrm{~cm}$; e $57 \%$ de argila, $6 \%$ de silte e $36 \%$ de areia no perfil de $20-40 \mathrm{~cm}$. 
A área de estudo tinha como principal uso a pastagem, com predominância de gramíneas das espécies Brachiaria brizantha Hochst Stapf (capim-braquiária) e Melinis minutiflora P. Beauv. (capim-gordura). Não houve registro de ocorrência de revolvimento intenso do solo à semelhança do que se utiliza nos cultivos agrícolas convencionais, no histórico de uso do solo nesse local. O espaçamento médio adotado no plantio mecanizado foi de 3,5 m entre as linhas.

\subsection{Coleta de dados}

O trator utilizado tem potência de $150 \mathrm{cv}$ turbinado, tração 4 x 2 auxiliar TDA, lastrado com $455 \mathrm{~kg}$ na parte dianteira e $150 \mathrm{~kg}$ em cada roda traseira e com peso total aproximado de $6.265 \mathrm{~kg}$. A bitola foi usada no máximo, com 2,04 $\mathrm{m}$ e largura total do rodado foi de 2,50 m.

Utilizou-se um subsolador-adubador de arraste com haste única lisa parabólica e ponteira não alada, com dimensões de $1 \frac{1}{1 / 2}$ polegadas de espessura e $32 \mathrm{~cm}$ de largura. Na área florestal, apesar de haver recomendações no sentido de que a profundidade de subsolagem seja proporcional de 5 a 7 vezes a largura da ponteira do subsolador (SPOOR; GODWIN, 1978 apud SASSAKI et al., 2005), essa profundidade é recomendada em função das características do sistema radicular do eucalipto.

O implemento foi tracionado por um trator com motor de potência bruta de aproximadamente $112 \mathrm{~kW}$, com tração dianteira auxiliar ativada e bitola regulada para a máxima largura entre rodas $(204 \mathrm{~cm})$, para maximizar o equilíbrio do conjunto em terrenos inclinados. Durante toda a operação, foi mantida velocidade em primeira marcha, obtendo $3,18 \mathrm{~km} / \mathrm{h}$, rendimento médio de $0,89 \mathrm{ha} / \mathrm{h}$ e eficiência operacional de 50,81\%.

A subsolagem foi realizada no sentido perpendicular à declividade do terreno, respeitando-se a inclinação lateral máxima permitida para o trator. Para as condições desse experimento, a inclinação lateral máxima foi calculada de acordo com o centro de gravidade e a bitola do trator (Equação 1), extraída de Mialhe (1980) e utilizada por Lima et al. (2004).

$$
i=\tan ^{-1} \frac{S}{2 y} \times f
$$

Em que: $\mathrm{i}=$ limite de inclinação do plano de apoio, graus; $\mathrm{S}=$ a bitola do trator, $\mathrm{mm} ; \mathrm{y}=$ coordenada vertical do centro de gravidade, $\mathrm{mm}$; e $\mathrm{f}=$ fator de segurança, decimal.
Na Equação 1, ilustra-se a condição de equilíbrio estático lateral do trator usado na operação, quando a inclinação do plano de apoio é igual a "i”. Excedido esse valor, teoricamente, o trator tomba. $\mathrm{O}$ fator de segurança sugerido é de 50\%, conforme descrito em Mialhe (1980).

Depois, foi medida, com o uso de um trado graduado, a profundidade que a haste de subsolagem efetivamente atingiu ao trabalhar no local, a cada 5 metros, mapeada com GPS geodésico GTR-1 da TechGeo.

Para efeito do estudo, consideraram-se como tratamentos, sete classes de declividade, que foram distribuídas de acordo com as variações do local, sendo 10,00 a $14,99 \% ; 15,00$ a $19,99 \% ; 20,00$ a $24,99 \% ; 25,00$ a 29,99\%; 30,00 a 34,99\%; 35,00 a 39,99\%; e 40,00 a 44,99\%.

Para a determinação do número e da amplitude das classes, foi utilizado o seguinte procedimento: obteve-se o número de classes necessárias pela fórmula de Sturges (Equação 2) e foi determinada a amplitude de classe (Equação 3):

$K \cong 1+3,22 \log (n) \cong 1+3,22 \log (91) \cong 7$

$h \cong \frac{A T}{K} \cong \frac{45-10}{7}=5$

Em que: $K=$ número de classes; $n=$ tamanho da amostra; $h=$ amplitude de classe; e $A T=$ amplitude total.

Os dados de profundidade de subsolagem obtidos em cada classe de declividade foram avaliados segundo a análise de regressão para o caso de dados com repetição a $5 \%$ de significância. Para isso, utilizaram-se 13 repetições em cada classe de declividade. $O$ procedimento utiliza um modelo de regressão linear $\left(Y=\beta_{0}+\beta_{1} X+\varepsilon\right)$ em função dos centros de classe de declividade (variável independente) e das profundidades obtidas para cada classe (variável dependente). A estimativa do parâmetro $\beta_{1}$ foi verificada segundo o teste $\mathrm{t}$ a fim de verificar a significância deste em relação ao modelo proposto.

Para verificar a validade do modelo e o valor das estimações foram calculados o coeficiente de determinação $\left(R^{2}\right)$ e a análise gráfica dos resíduos, respectivamente em função das Equações 4 e 5.

$R^{2}=\frac{\text { SQRegressão }}{\text { SQTratamento }}$

$\operatorname{Erro}(\%)=\frac{Y-\hat{Y}}{Y} 100$

Em que: $Y=$ profundidade real; e $\hat{Y}=$ profundidade estimada.

Cerne, Lavras, v. 18, n. 4, p. 607-612, out./dez. 2012 


\section{RESULTADOS E DISCUSSÃO}

Conhecendo os dados de coordenadas do centro de gravidade e da bitola do trator no estudo, foi determinado o limite da inclinação lateral, conforme a Equação 1:

$i=\tan ^{-1} \frac{2040}{2 \times 850} \times 0,5=25,1^{\circ}$

A inclinação lateral máxima encontrada para tráfego do trator foi de $25,1^{\circ}$ ou o equivalente a $46,84 \%$. Assim, o tráfego no local de estudo ficou limitado onde a declividade não excedeu a inclinação de $46,84 \%$, estando alinhado com recomendações de Lima et al. (2004). Esses autores observaram que a realização de algumas atividades no setor florestal, pode ser limitada em regiões de topografia acidentada, por não permitir o tráfego das máquinas com estabilidade.

O resultado da análise de variância da regressão para o caso de dados com repetição está descrito na Tabela 1.

De acordo com o resultado da Tabela 1, a falta de ajustamento foi não significativa. Isso garante que o modelo é adequado para descrever os dados e que o Quadrado Médio da Falta de Ajustamento (QMFA) não está estimando um erro sistemático além do valor da variância.

Ainda, de acordo com a Tabela 1, o modelo de regressão proposto foi adequado para descrever os valores uma vez que, para a regressão, o resultado da análise foi significativo para o teste F. Isso indicou uma relação entre as duas variáveis analisadas.

A partir do resultado da ANOVA (análise de variância), foi estimada a equação de regressão linear e o coeficiente de determinação conforme Tabela 2.

$\mathrm{O}$ ajuste da equação de regressão indicou que o modelo é adequado uma vez que o coeficiente de regressão associado à declividade teve resultado significativo pelo teste t. Para esse ajuste, o coeficiente de determinação, utilizando-se a Equação 2, apresentou como resultado $78,95 \%$, indicando a quantidade de valores de profundidade que são explicados pela declividade.

De acordo com os valores das estimativas dos parâmetros da equação ajustada, obteve-se a amplitude das profundidades de subsolagem alcançadas em cada classe de declividade (Tabela 3).

Como a profundidade de subsolagem mínima recomendada é de 0,50 m no preparo do solo, em áreas mecanizáveis, para plantio de eucalipto, abaixo de $35,00 \%$ de declividade, atingiu-se a profundidade mínima esperada para revolvimento das camadas compactadas do povoamento de eucalipto. Na classe de declividade de 35,00 a $39,99 \%$ ocorre a faixa limite de profundidade de subsolagem recomendada. Acima de $40,00 \%$, não se recomenda a subsolagem, pois não se atinge a profundidade mínima esperada (Tabela 3 ).

Tabela 1 - Análise de variância para o caso de dados com repetição.

Table 1 - Variance analysis for the the case of data with repetition.

\begin{tabular}{lcccc}
\hline FV & GL & SQ & QM & Fcal \\
\hline Regressão & 1 & 2059,84 & 2059,84 & $34,66^{*}$ \\
Falta ajustamento & 5 & 549,35 & 109,87 & $1,85^{\mathrm{ns}}$ \\
(Tratamento) & 6 & 2609,19 & 434,87 & \\
Resíduo Puro & 84 & 4991,37 & 59,42 & \\
\hline Total & 90 & 7600,56 & & \\
\hline
\end{tabular}

Notas: *significativo ao nível de $5 \%$ de probabilidade

${ }^{\mathrm{ns}}$ não significativo a $5 \%$ de probabilidade

Tabela 2 - Equação linear com o respectivo coeficiente de determinação.

Table 2 - Linear equation with its coefficient of determination.

\begin{tabular}{cc}
\hline Equação linear * & Coeficiente de Determinação $\left(\mathrm{R}^{2}\right)$ \\
\hline$P R O F=67,99-0,4758 D E C$ & $\mathrm{R}^{2}=78,95 \%$ \\
\hline
\end{tabular}

Notas: *significativo a $5 \%$ de probabilidade pelo teste $\mathrm{t}$

$\mathrm{PROF}=$ profundidade obtida nas classes de declividade; e DEC = classes de declividade.

Cerne, Lavras, v. 18, n. 4, p. 607-612, out./dez. 2012 
Tabela 3 - Amplitude das profundidades de subsolagem em cada classe de declividade.

Table 3 - Range of the depths of subsoiling in each class of slope.

\begin{tabular}{ccc}
\hline Classes de declividade & \multicolumn{2}{c}{ Profundidades de subsolagem $(\mathrm{m})$} \\
\cline { 2 - 3 }$(\%)$ & Mínimas & Máximas \\
\hline $10,00-14,99$ & 0,61 & 0,63 \\
$15,00-19,99$ & 0,59 & 0,61 \\
$20,00-24,99$ & 0,56 & 0,59 \\
$25,00-29,99$ & 0,54 & 0,56 \\
$30,00-34,99$ & 0,51 & 0,54 \\
$35,00-39,99$ & 0,49 & 0,51 \\
$40,00-44,99$ & 0,47 & 0,49 \\
\hline
\end{tabular}

Para Fessel (2003) e Srivastava et al. (1993), a subsolagem é utilizada para romper camadas de solo impermeáveis ou compactadas, em profundidades maiores que $40 \mathrm{~cm}$, promovendo a infiltração de água, pouca mistura e nenhuma inversão de solo. Nesse sentido, respeitandose a declividade máxima para a subsolagem de $46,84 \%$, atingiu-se o objetivo de preparo mecanizado de solo, com profundidade mínima de $0,50 \mathrm{~m}$, até o limite de $40 \%$ de declividade com segurança e eficiência.

Ainda para verificar o comportamento da equação ajustada foi realizada uma análise gráfica de resíduos, utilizando-se a Equação 5, a fim de melhor estudar o efeito da profundidade nas diferentes classes de declividade, conforme mostrado na Figura 1.

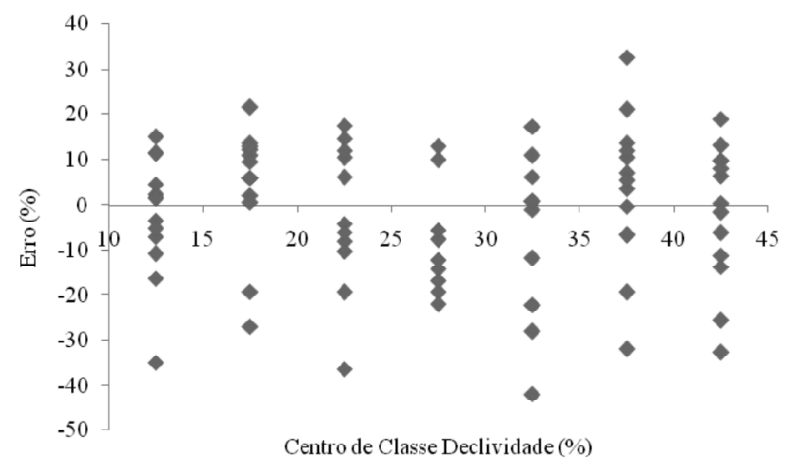

Figura 1 - Análise gráfica de resíduos.

Figure 1 - Graphical analysis of residues.

De acordo com os resultados dos resíduos apresentados na Figura 3, pode-se verificar que para os centros de classe de 12,$5 ; 22,5 ; 27,5$ e 42,5 houve uma superestimação dos valores de profundidade em relação aos centros de classe de 17,5 e 37,5 em que houve subestimação dos valores. Na classe de 37,5, houve uma igualdade entre as superestimações e subestimações, porém as subestimações foram mais discrepantes em relação ao valor original, gerando um erro de maior magnitude.

Esses resultados, nas condições do trabalho, são condizentes com as orientações técnicas de empresas florestais de referência (DALBEM et al., 2008), em relação à profundidade de trabalho.

\section{CONCLUSÕES}

A profundidade da subsolagem diminui com o aumento da declividade do terreno.

O modelo linear propostoé adequado para descrever a relação de que $78,95 \%$ dos valores de profundidade foram explicados pelos centros de classe das declividades.

O ajuste da equação de regressão indica que o modelo é adequado uma vez que o coeficiente de regressão associado à declividade teve resultado significativo pelo teste $\mathrm{t}$, a $5 \%$ de probabilidade.

A declividade do terreno, sendo um limitador para a qualidade da operação de preparo mecanizado do solo, demonstra que declividades até $40 \%$ permitem atingir a profundidade mínima de $0,50 \mathrm{~m}$ para a subsolagem.

\section{REFERÊNCIAS}

BLAZIER, M. A.; DUNN, M. Stock type, subsoiling, and density impact productivity and land value of a droughty site. Southern Journal of Applied Forestry, Washington, v. 32, n. 4, p. 154-162, 2008.

CHISHOLM, C. J. A mathematical model of tractor overturning and impact behavior. Journal of Agricultural Engineering Research, London, v. 24, p. 375-394, 1979.

DALBEM, M.; DAL'COL, J.; SARCINELLI, T. S.; CARMO, A. P. do. Fomento legal: cultivando o eucalipto. Aracruz: Pauta 6, 2008. 50 p.

DEDECEK, R. A.; CURCIO, G. R.; RACHWAL, M. F. G.; SIMON, A. A. Effects of soil tillage systems on soil erosion and on black wattle (Acacia mearnsii De Wild.) productivity. Ciência Florestal, Santa Maria, v. 17, n. 3/4, p. 205-215, 2007.

Cerne, Lavras, v. 18, n. 4, p. 607-612, out./dez. 2012 
FESSEL, V. A. G. Qualidade, desempenho operacional e custo de plantios, manual e mecanizado, de Eucalyptus grandis, implantados com cultivo mínimo. 2003. 88 p. Dissertação (Mestrado em Ciências Florestais) - Escola Superior de Agricultura "Luiz de Queiroz", Piracicaba, 2003.

GATTO, A.; BARROS, N. F. de; NOVAIS, R. F. de; COSTA, L. M. da; NEVES, J. C. L. Efeito do método de preparo do solo, em área de reforma, nas suas características, na composição mineral e na produtividade de plantações de Eucalyptus grandis. Revista Árvore, Viçosa, v. 27, n. 5, p. 635-646, 2003.

GIALAMAS, T. A.; BOCHTIS, D.; AMPATZIDIS, Y.; KATERIS, D.; GEMTOS, T. A.; TSATSARELIS, C. A. Tractor stability estimation using adjusting testing-bench. In: THE INTERNATIONAL CONFERENCE HAICTA, 2006, Volos. Proceedings... Volos: 2006. p. 179-186.

GILFILLAN, G. Attitude of a tractor on sloping land. Journal of Agricultural Engineering Research, London, v. 12 , n. 4 , p. 293-296, 1967.

GONÇALVES, J. L. M.; STAPE, J. L.; BENEDETTI, V.; FESSEL, V. A. G.; GAVA, J. L. Reflexos do cultivo mínimo e intensivo do solo em sua fertilidade e na nutrição das árvores. In: GONÇALVES, J. L. M.; BENEDETTI, V. (Ed.). Nutrição e fertilização florestal. Piracicaba: IPEF, 2000. p. 1-57.

HUNTER, A. G. M. Tractor safety on slopes. Agricultural Manpower, Wallingford, v. 36, n. 4, p. 95-98, 1981.

LANI, J. L. (Coord.). Atlas de ecossistemas do Espírito Santo. Vitória: SEMA; Viçosa, MG: UFV, 2008. 504 p.

LIMA, J. S. S.; SOUZA, A. P.; MACHADO, C. C. Estimativa das estabilidades longitudinal e transversal de tratores florestais utilizados na colheita de madeira. Revista Árvore, Viçosa, v. 28, n. 6, p. 839-844, 2004.

MASHADI, B.; NASROLAHI, H. Automatic control of a modified tractor to work on steep side slopes. Journal of Terramechanics, Oxford, v. 46, p. 299-311, 2009.

MIALHE, L. G. Máquinas motoras na agricultura. São Paulo: Pedagógica e Universitária, 1980. v. 2, 367 p.

Cerne, Lavras, v. 18, n. 4, p. 607-612, out./dez. 2012
MINATEL, A. L. G.; ANDRIOLI, I.; CENTURION, J. F; NATALE, W. Efeitos da subsolagem e da adubação verde nas propriedades físicas do solo em pomar de citros. Engenharia Agrícola, Jaboticabal, v. 26, n. 1, p. 86-95, 2006.

MOLIN, J. P.; SILVA JÚNIOR, R. L. da. Variabilidade espacial do índice de cone, correlacionada com textura e produtividade. Engenharia Rural, Piracicaba, v. 14, p. 49-58, 2003.

PEREIRA, D. P. Análise técnica e de custos de povoamento de eucalipto sob preparo manual e mecanizado do solo em área declivosa no sul do Espírito Santo. 2010. 112 f. Dissertação (Mestrado em Ciências Florestais) - Universidade Federal do Espírito Santo, Alegre, 2010.

RAPER, R. L.; BERGTOLD, J. S. In-row subsoiling: a review and suggestions for reducing cost of this conservation tillage operation. Applied Engineering in Agriculture, Saint Joseph, v. 23, n. 4, p. 463-471, 2007.

RAPER, R. L.; SCHWAB, E. B.; BERGTOLD, J. S.; PRICE, A. J.; BALKCOM, K. S.; ARRIAGA, F. J.; KORNECKI, T. $\mathrm{S}$. Maximizing cotton production and rye cover crop biomass through timely in-row subsoiling. Applied Engineering in Agriculture, Saint Joseph, v. 25, n. 3, p. 321-328, 2009.

RICHART, A.; TAVARES FILHO, J.; BRITO, O. R.; LLANILLO, R. F.; FERREIRA, R. Compactação do solo: causas e efeitos. Semina, Passo Fundo, v. 26, n. 3, p. 321-344, 2005.

SALVADOR, N.; BENEZ, S. H.; MION, R. L. Consumo de combustível na operação de subsolagem realizada antes e depois de diferentes sistemas de preparo periódico do solo. Engenharia Agrícola, Jaboticabal, v. 28, n. 2, p. 256-262, 2008.

SASSAKI, C. M.; GONÇALVES, J. L. M.; BENTIVENHA, S. R. P. Desempenho operacional de hastes subsoladoras em função da ponteira e do tipo do solo. Scientia Forestalis, Piracicaba, n. 67, p. 44-52, 2005.

SPENCER, H. B. Stability and control of two-wheel drive tractors and machinery on sloping ground. Journal of Agricultural Engineering Research, London, v. 23, p. 169-188, 1978.

SRIVASTAVA, A. K.; GOERING, C. E.; ROHRBACH, R. P. (Ed.). Engineering principles of agricultural machines. Saint Joseph: American Society of Agricultural Engineers, 1993. $601 \mathrm{p}$. 